\title{
Quadcopter based emergency medikit delivery system for hill stations
}

\author{
C. R. Balamurugan', S. M. Revathy ${ }^{2}$, P. Vijayakumar ${ }^{3}$ \\ ${ }^{1,3}$ Department of EEE, Karpagam College of Engineering, India \\ ${ }^{2}$ Department of ECE, University College of Engineering, India
}

\begin{abstract}
Article Info
Article history:

Received Dec 12, 2019

Revised Jan 12, 2020

Accepted Mar 19, 2020

Keywords:

Arduino

GPS

PWM

Quadcopter

Wireless camera

ABSTRACT

Now-a-days UAV's are more common for search, rescue and surveillance. A quad copter UAV system is proposed to be used for aerial transportation of medicine. A Quadcopter has a drive chassis having a propeller, ESC, motor, frames and battery. Quadcopter is supported with GPS to know the exact position. To know the exact location and delivering medicine to accident area, an audio and video system is used. The quad frame built from quality materials, which are reinforced and much stronger, this reduces arm breakage. A set of two plastic propellers, one normal and one pusher (reverse) to rotate and lift up the quadcopter. The brushless out-runner will provide more power with its high efficiency, long run times. The ESC includes programmable motor braking, soft start for helicopters and planes, timing, throttle input range and low-voltage cutoff. Lithium-Polymer (Li-Po) battery for very light weight, small size and durability without losing charging capacity. The whole quadcopter process can be monitored and controlled by a remote control system, quadcopter will capture the live video and current status can be seen visualized and provides information about all the other exact condition in real time.
\end{abstract}

This is an open access article under the CC BY-SA license.

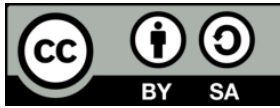

\section{Corresponding Author:}

C. R. Balamurugan,

Department of EEE,

Karpagam College of Engineering,

Myleripalayam Village, Othakkal Mandapam Post, Coimbatore - 641032, Tamilnadu, India.

Email: hod.eee@kce.ac.in

\section{INTRODUCTION}

This investigation is in the fields of Embedded System. Embedded System is defined as the combination of Hardware and Software, to perform a particular task in a dedicated manner. The major area of this paper is [1] robotics. The implementation of this paper is to transport the Medicine to the accidental area as soon as possible. In present days the transportation of medicine by road is very difficult due to the vehicles get stuck in traffic, while an accident occurs and this prohibits the delivery of emergency medicine to the injured person. For this transportation an UAV is employed. In UAV, the controlling and operation of quadcopter is usually done by using RF [Radio Frequency] circuits. This circuits are widely used for control and working applications and are also reliable over a range. The RF circuit consist of transmitter and receiver which are dependent of each other. All the control signal and commands are sent via wireless medium in between transmitter and receiver. "Necessities are the mother of inventions", whenever human being finds the need of something it will lead to a wonderful invention. Quadcopter is one of the wonderful invention in the field of robotics [2]. 
Quadcopters differ from conventional helicopters which use rotors which are able to vary the pitch of their blades dynamically as they move around the rotor hub. In the early days of flight, quadcopters (then referred to either as 'quadrotors' or 'helicopters') were seen as possible solutions to some of the persistent problems in vertical flight; torque-induced control issues [3] (as well as efficiency issues originating from the tail rotor, which generates no useful lift) can be eliminated by counter-rotation and the relatively short blades are much easier to construct. A number of manned designs appeared in the 1920s and 1930s. These vehicles were among the first successful heavier-than-air vertical take-off and landing (VTOL) vehicles. However, early prototypes suffered from poor performance, and latter prototypes required too much pilot work load, due to poor stability augmentation and limited control authority.

Medicine transportation in the Peruvian Amazon Rainforest is difficult due to environmental hazards and lack of proper land transportation roads, which leads to insufficient and improper health services in this area. In order to overcome the terrestrial impediments mentioned above and remedy this situation, a quadcopter UAV system is proposed to be used for aerial transportation of medicine. This paper will explain the mechanical, electrical and control design considerations taken into account for the implementation of the proposed system. Quadcopter frame modeling is useful to analyze the reliability of body frame part and to help determine the type of rotor and propeller in order to assure the necessary flight acceleration. Quadcopter flight stability is influenced by the resulting thrust, by the distance between each rotor propeller and also by the frame rigidity; the frame has been designed to be as light as possible, meanwhile maintaining the strength to carry the load. Solidworks software has been used to design and analyze using Finite Element Analysis (FEA) method the quadcopter frame, having folded size of 560 (mm) square,also a 406×127 (mm) propeller and rotor's angular velocity and air flow produced around the propeller. The FEA method showed that the presence of rotational velocities in each propeller flow field will significantly affect the thrust efficiency which can cause flight instability or body frame vibration. Mine detection using a surveillance drone is a modern conceptual prototype, which has been designed to detect landmines. Landmines were primarily used to create defensive and tactical barriers during the Second World War. They are still very much employed in large quantities in countries such as Afghanistan, Korea. A lot of these land mines still go undetected, increasing the death rate and creating havoc on the surroundings. The prototype developed helps us to detect a landmine using a flying drone. The prototype has a quad copter which has a mine detector mounted on it. This utilizes two different modes of detection, which are an IR camera and a metal detector. These are extensively used in aiding this whole operation. The location of the mine can be traced back by the GPS and the detected location can be transmitted using the GSM module. There is a lot of untapped potential and scope of improvement for this prototype in the future. A rotorcraft UAV is any flying machine that produces lift from rotors turning in a plane that is normally close to the horizontal. The task of building a rotorcraft UAV platform for academic research may be daunting if the enormous challenges and constraints associated with the research are considered. It involves design and construction of the vehicle, and careful consideration of factors such as hardware components selection, layout design, desired range and performance evaluation, among others. Although, there exist some commercial-of-the-shelf (COTS) platforms such as Draganfly, Microdrones [4], Arducopter, etc., that can be easily adopted.

However, it is difficult to find a platform that effectively meets all the intended study requirements. Therefore, in such a situation, it becomes necessary to build a platform that suits the research objectives. The design, construction and kitting of a rotorcraft UAV comprises the following five key steps: (i) virtual design environment selection (ii) hardware components selection [5] (iii) avionic system design and integration (iv) vehicle design and construction (v) performance and reliability evaluation. This paper presents a systematic design methodology for the construction of a small-scale rotorcraft UAV system. For the delivery of medicines, ambulances (four and two wheelers) are employed which may get stuck in traffic when an accident occurs. So the transportation of medikit is done by the quadcopter system to avoid the human losses.

\section{OVERVIEW}

The UAV industry has seen increased technological developments in recent years, thus creating more accessible flying autonomous vehicles. With it, companies around the world identified commercial applications for said technology, namely as low-cost transportation of low-weight cargo vehicles. Companies such as Google, Amazon and DHL have developed their own prototypes with the goal of creating commercial networks based around UAV transportation. Other companies have focused on more humanitarian applications. To develop a combine medicine delivery system using quadcopter with audio video transmitting system and a global positioning system. This system can deliver the medicine for the people at risk in accidental area and the places where the medicine is not delivered by roadways (tribal 
areas). The setup of this quadcopter model is emphasized with the gyro and accelerometer sensor for auto correcting the deviation that occurs during the flight. Without using the other costlier flight control boards, arduino uno board is used. To know the exact position of the quadcopter, GPS system is fixed in quadcopter using GSM technologies for tracking over the web.

This paper is to design a quadcopter for medicine delivery purpose. This paper is very much useful in accidental area for first aid for injured person. It is much more useful for saving a human life which is at risk. The implementation of this paper is done with a wireless controlled quadcopter with video support that completely controlled with wireless system.Quadcopters differ from conventional helicopters which use rotors which are able to vary the pitch of their blades dynamically as they move around the rotor hub.

In the early days of flight, quadcopters (then referred to either as 'quadrotors' or 'helicopters') were seen as possible solutions to some of the persistent problems in vertical flight,torque-induced control issues (as well as efficiency issues originating from the tail rotor, which generates no useful lift) can be eliminated by counter-rotation and the relatively short blades are much easier to construct.A number of manned designs appeared in the 1920s and 1930s. These vehicles were among the first successful heavier-than-air vehicles. However, early prototypes suffered from poor performance, and latter prototypes required too much pilot work load, due to poor stability augmentation and limited control authority. Quadcopters are designed with expansion in mind. The flat and symmetrical frame makes it easy to incorporate more hardware onto the design. They have self-balancing abilities that allow for minor frame imbalance, which enables hardware to be added without the concern for perfect balance.

\section{BLOCK DIAGRAM}

Block diagram of overall system hardware as illustrated on Figure 1 and Figure 2 show the Circuit connection diagram.

- Wireless Transmitter: For controlling the quadcopter and to provide the control signal from the user.

- Base Station Computer: To receive the GPS signal and audio video signals.

- Wireless Receiver: Receiving the control signal from the transmitter for the control action of UAV.

- Flight controller: To produce the control signal for increasing the voltage in step by step manner.

- ESC: To vary the voltage supplying to the motor.

- Motor: To produce the rotating force for uplift of the UAV.

- Sensors: To balance the quadcopter.

- GPS: Determination of exact position of the quadcopter is done.

- Audio and video: Audio and video signals are transmitted.

- Transmitter

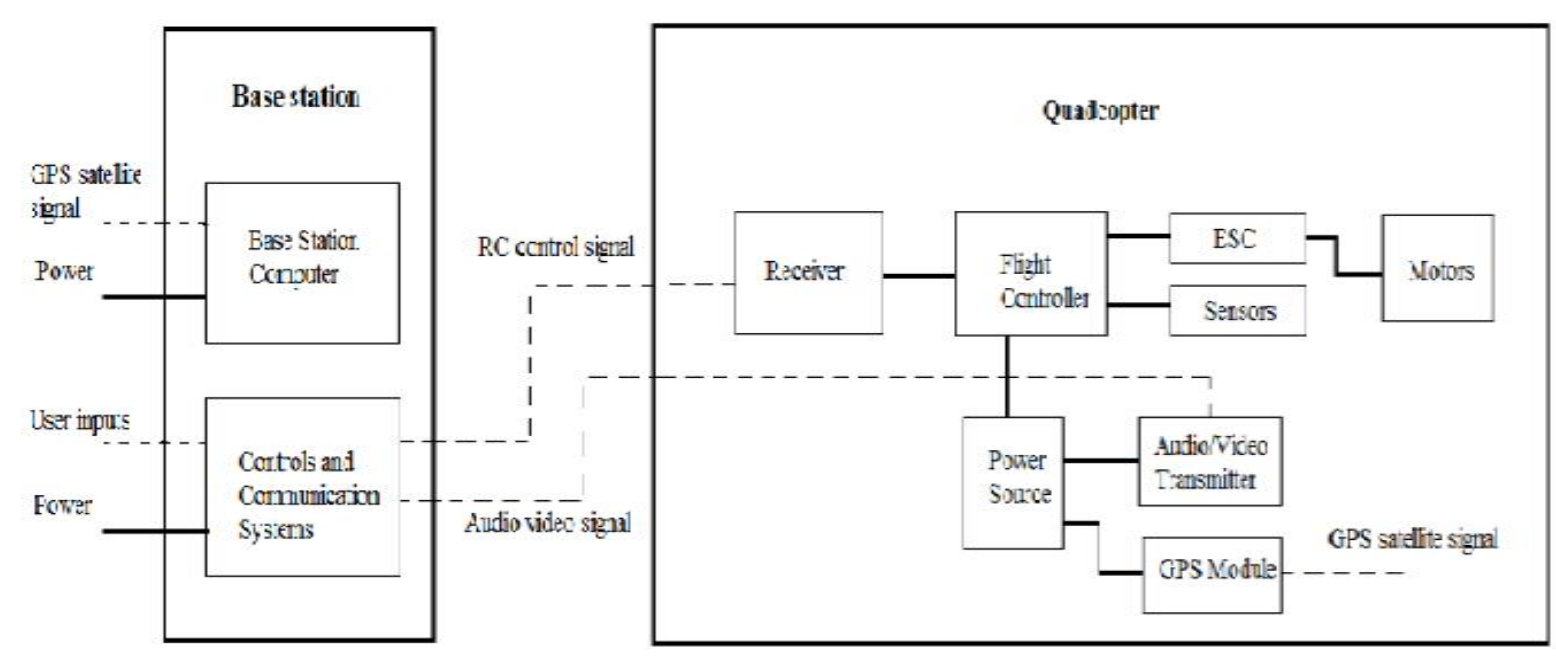

Figure 1. Block diagram of overall system hardware 


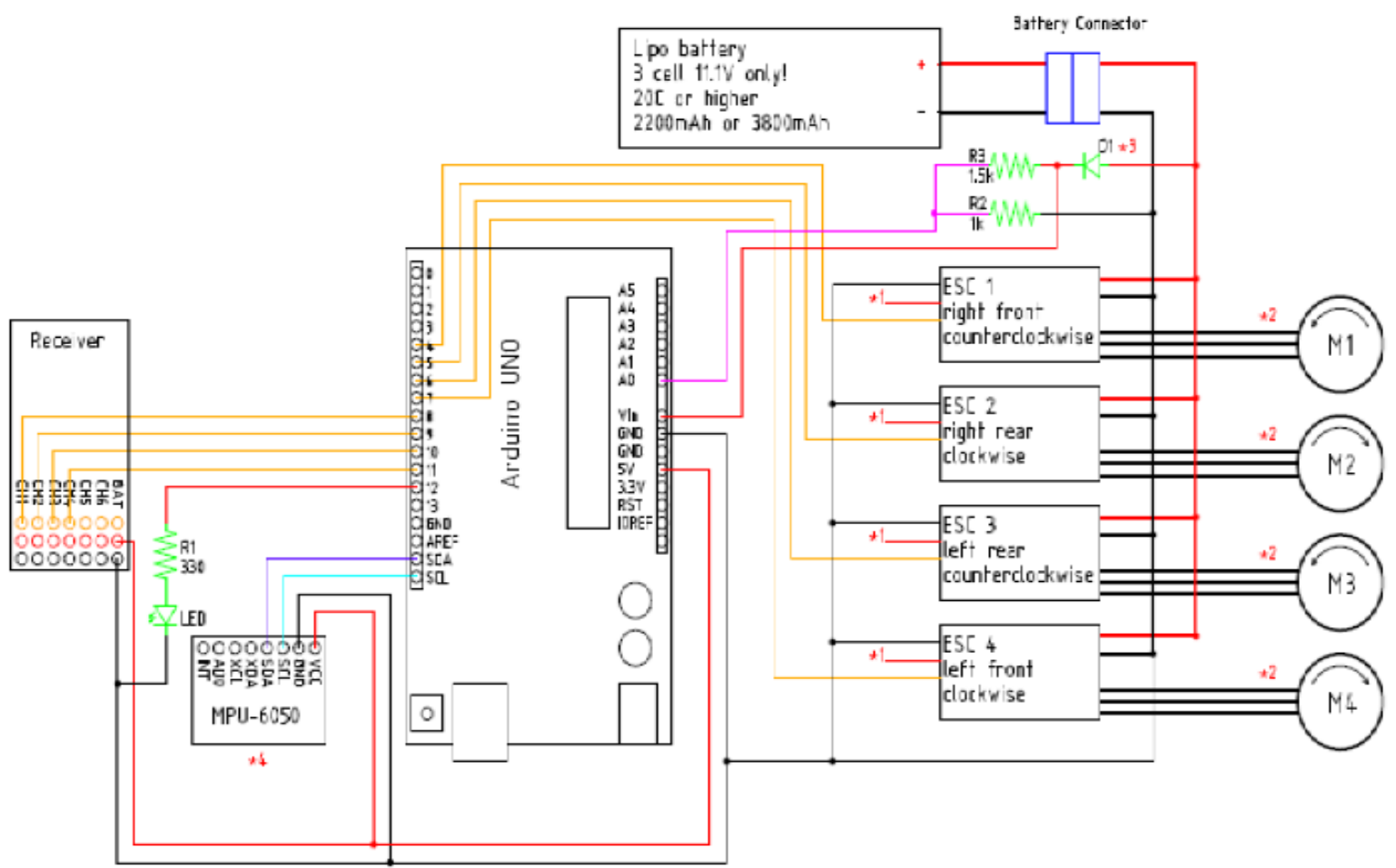

Figure 2. Circuit connection diagram

\section{HARDWARE DESCRIPTION}

Figure 3 display the quadcopter frame. The frame of the quadcopter provides the physical structure for the entire aircraft. It joins the motors to the rest of the aircraft and houses all of the other components. The frame must be large enough to allow all four propellers to spin without collision, but must not be too large and therefore too heavy for the motors. In selecting quadcopter body frame, one must regard the total weight which will be borne by quadcopter such as the weight of electronic, frame, landing gear, rotor and sensor. For this model, the size was determined firstly so that the type of rotor and propeller used can be calculated in terms of quadcopter ability to carry weight. The frame shall be strong enough to carry the components necessary to accomplish this work and be able to withstand the stress caused during landing. Many quadcopter frames are available: CF-04, ZMR180 CARBON FIBER, LISAM LS-210, HS250 CARBON FIBER, DIATONE Q450 QUAD FRAME. Among these frames each possesses its own advantages, here DIATONE Q450 QUAD FRAME is selected for its below advantages: This frame is built by high quality and ultra-durable materials, by which the main frame is constructed in high quality glass fiber, while arms are constructed from ultra-durable polyamide nylon. Quadcopter frames are reinforced and much stronger, this reduces arm breakage. Additional advantage is, mainframe bottom plate is provided for the place for mounting cameras or other accessories. Also, it has integrated PCB connections for soldering ESC's and colored arms for the identification, orientation of the quadcopter while flying. Pre threaded brass sleeves for all of the frame bolts. The weight and size of the frame is 295 grams and $450 \mathrm{~mm}$ respectively.

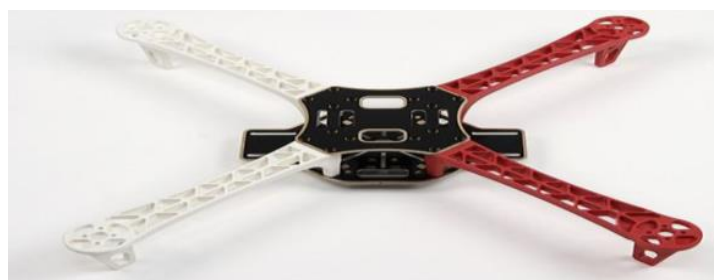

Figure 3. Quadcopter frame 
A brushless electric motor is an electric motor driven by an electrical input, which lacks any form of commutator or slip ring. The motor requires some form of alternating current to turn, either from an AC supply, or an electronic circuit. A brushless DC electric motor contains synchronous motor and integrated power electronics that operate the motor from a direct current supply. A "permanent-magnet synchronous motor" (PMSM) or "permanent-magnet motor" (PMM) is a synchronous motor that uses permanent magnets rather than windings in the rotor. Electronic excitation control with integrated power inverter and rectifier, sensor, and inverter electronics is required for practical operation. Form-factors for the PMSMs are either axial flux, radial flux, transverse flux, or flux switching depending on the arrangement of components, with each topology having different tradeoffs among efficiency, size, weight, and operating speed. Also in the axial flux or radial flux form-factor, other brushless motors are the brushless wound-rotor doubly-fed synchronous motor system and the magnetic reluctance, which require electronic excitation control for practical operation, and the asynchronous induction motors, which is with or without electronic excitation control depending on the application. Conventional methods of electronic AC excitation control of electric motors, such as volt/frequency control, were available prior to the $1950 \mathrm{~s}$ with at least mercury vapor thyratrons switching devices and modern age state of art control (or Field Oriented Control) became available in the late 1960s. Permanent magnet motors have been in existence since the introduction of electric motors more than one-hundred years ago.

For the selection of motors two considerations are to be done: (i). Quadcopter planned total weight, and (ii) Size of frame. Total weights includes the frame, motor, ESC, propeller, flight control board and payload weights. By knowing these things, we can roughly calculate how much thrust the motors need to deliver to lift the quadcopter in the air.

A general rule is that it should at least be able to provide twice as much thrust than the weight of the quad. This is the bare minimum to ensure a stable copter that is easy to control during hovering. If the thrust provided by the motors are too little, the quad would not respond well to control, it might even have difficulties to take off. For the fast flying, the thrust: weight ratio is to be $3: 1$. Brushless motors in RC is indicated by a 4-digit number - AABB. "AA" the stator width, and "BB" the stator height. Basically, the wider and taller the motor is the more torque can be generate. KV is another important parameter, it's the theoretical increase of motor RPM (rotation per minute) when voltage go up by 1 volt without load. But once a propeller is mounted on the motor, the RPM won't be that high due to resistance. Higher KV motors would attempt to spin the propeller faster, but lower KV motors normally generate higher torque. That's why the larger props are paired with low KV motors, and smaller props with high KV motors. It's important to find a balance between RPM and torque when choosing motor and propeller. By pairing high KV motors with excessively large propellers, the motor will attempt to spin it fast like they would do with smaller props, and therefore drawing too much current and generating too much heat. Eventually it could burn out the motor due to overheat and shorts in motor coils. Figure 4 shows the brushless out runner motor.

On motors sometimes see something like "12N14P". The number before the letter $\mathrm{N}$ means the number of electromagents in stator, and the number before $\mathrm{P}$ means the number of permanent magnets in the motor. Most motors have the same 12N14P configuration, some lower KV motors would have more electromagnets and permanent magnets to help increase torque more efficiently (and thus more expensive). While it's good to know what this is, it's not an essential piece of information when picking motors. If the motor draws high current, the battery is able to supply for that, if not then the battery discharge is affected. $\mathrm{C}$ ratings are to be considered for the battery capacity.

\author{
Motor Specifications \\ 1. KV (rpm/volt): 1400 \\ 2. Thrust (g/s): 1265/4 \\ 3. Efficiency: $80 \%$ \\ 4. Efficiency current: 4-10A (>75\%) \\ 5. Current Capacity: $12 \mathrm{~A} / 60 \mathrm{~s}$ \\ 6. No Load current @10V: 0.5A \\ 7. Working voltage range: $7-12 \mathrm{v}$ \\ 8. Shaft Diameter: $3.17 \mathrm{~mm}$
}




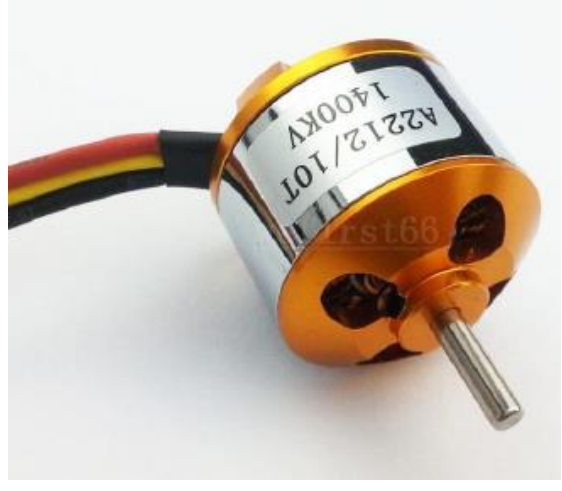

Figure 4. Brushless out runner motor

Basically, the difference of $\mathrm{CW}$ and $\mathrm{CCW}$ motor is the prop shaft thread rotation. The intention is to use $2 \mathrm{CW}$ motors and $2 \mathrm{CCW}$ motors on a quad, so that when the motors spin, all four prop nuts lock themselves down. They are to be identical motors except the prop shaft thread. The preference to get all motors of the same threads so there is no confusion with the different prop nuts. Propellers exist in different length and pitch. The length of a propeller is the diameter of a disc the prop makes when it's spinning. Pitch can be defined as the travel distance of one single prop rotation. It's sometimes called pitch length too. The larger the prop (either increasing diameter, or pitch or both), the harder it's going to spin. Propellers generate thrust by spinning and moving air. The more air it moves, the more thrust it generates. By increasing either prop length or pitch, it gives more thrust and leads to higher current draw. Effectively it increases the surface area and drag, so more air can be moved and more power is required to spin it. In a nutshell, larger propeller or higher pitch length will increase your aircraft's speed but also use more power.

A prop with smaller diameter or pitch can spin faster (higher RPM), because the motor doesn't need to work as hard to spin it so it pulls less current. They tend to run smoother and feels more responsive to the sticks. The faster change of RPM due to less inertia helps stability of the quadcopter.

A higher pitch propeller moves greater amount of air, which might create turbulence and cause more prop wash. Props spin slower and you might find the lack of "snappy" feeling. But it generates more thrust and higher top speed in the expense of higher current draw. The more surface area, the more air the propeller can move and thus generate more thrust. But the downside is higher current draw, more drag and drop in power efficiency. Figure 5 displays the propellers. The shape of a propeller plays a big role in performance because it's closely related to surface area. The most distinctive difference would probably be the tip of the props: pointy nose, bull nose $(\mathrm{BN})$ and Hybrid bullnose $(\mathrm{HBN})$. HBN has more surface area than pointy nose, while BN has even more surface area than the HBN. Pointy tip props are the most efficient of the three, however it pulls the least thrust. The other two props can give more power in the cost of more current draw. The shape of the tip also changes how the prop interacts with airflow when spinning, props with smooth/round edges perform more efficiently than blunt/square tip props. On every quadcopter, there are two CW (clockwise) and two CCW (counter-clockwise) propellers. CW and CCW props are supposed to rotate at the opposite direction to generate thrust, as well as having opposing yaw motion that cancel each other out in flight.

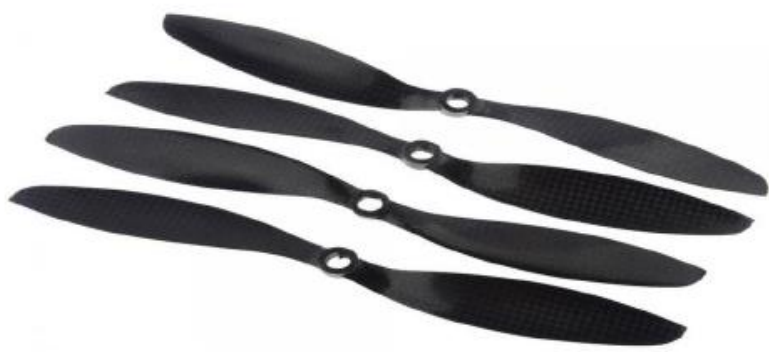

Figure 5. Propellers 
Table 1. Propellers specifications

\begin{tabular}{lllll}
\hline \multicolumn{1}{c}{ Frame size } & \multicolumn{1}{c}{ Propeller size } & \multicolumn{1}{c}{ Motor size } & \multicolumn{1}{c}{ KV } & Frame size \\
\hline $150 \mathrm{~mm}$ or smaller & 3" or smaller & 1306 or smaller & $3000 \mathrm{KV}$ or higher & $150 \mathrm{~mm}$ or smaller \\
$180 \mathrm{~mm}$ & $4 "$ & 1806 & $2600 \mathrm{kv}$ & $180 \mathrm{~mm}$ \\
$210 \mathrm{~mm}$ & $5 "$ & $2204-2206$ & $2300-2600 \mathrm{kv}$ & $210 \mathrm{~mm}$ \\
$250 \mathrm{~mm}$ & $6 "$ & $2204-2208$ & $2000-2300 \mathrm{kv}$ & $250 \mathrm{~mm}$ \\
$350 \mathrm{~mm}$ & $7 "$ & 2208 & $1600 \mathrm{kv}$ & $350 \mathrm{~mm}$ \\
$450 \mathrm{~mm}$ & $8,, 9 ", 10 "$ & 2212 or larger & 1000 or smaller & $450 \mathrm{~mm}$ \\
\hline
\end{tabular}

An electronic speed control or ESC is an electronic circuit with the purpose to vary an electric motor's speed, its direction and possibly also to act as a dynamic brake. ESCs are often used on electrically powered radio-controlled models, with the variety most often used for brushless motors essentially providing an electronically generated three-phase electric power low voltage source of energy for the motor. An ESC can be a stand-alone unit which plugs into the receiver's throttle control channel or incorporated into the receiver itself, as is the case in most toy-grade $\mathrm{R} / \mathrm{C}$ vehicles. Some $\mathrm{R} / \mathrm{C}$ manufacturers that install proprietary hobby-grade electronics in their entry-level vehicles, vessels or aircraft use onboard electronics that combine the two on a single circuit board.

Regardless of the type used, an ESC interprets control information not as mechanical motion as would be the case of a servo, but rather in a way that varies the switching rate of a network of field effect transistors, or FETs. The rapid switching of the transistors is what causes the motor itself to emit its characteristic high-pitched whine, especially noticeable at lower speeds. It also allows much smoother and more precise variation of motor speed in a far more efficient manner than the mechanical type with a resistive coil and moving arm once in common use. Most modern ESCs incorporate a battery eliminator circuit (or BEC) to regulate voltage for the receiver, removing the need for separate receiver batteries. BECs are usually either linear or switched mode. ESCs, in a broader sense, are PWM controllers for electric motors. The ESC generally accepts a nominal $50 \mathrm{~Hz}$ PWM servo input signal whose pulse width varies from $1 \mathrm{~ms}$ to $2 \mathrm{~ms}$. When supplied with a $1 \mathrm{~ms}$ width pulse at $50 \mathrm{~Hz}$, the ESC responds by turning off the motor attached to its output. A $1.5 \mathrm{~ms}$ pulse-width input signal drives the motor at approximately half-speed. When presented with $2.0 \mathrm{~ms}$ input signal, the motor runs at full speed. ESC systems for brushed motors are very different by design; as a result brushed ESC's are not compatible with brushless motors. Brushless ESC systems basically create a tri-phase AC power output of limited voltage from an onboard DC power input, to run brushless motors by sending a sequence of AC signals generated from the ESC's circuitry, employing a very low impedance for rotation. Brushless motors, otherwise called out-runners or in-runners depending on their physical configuration, have become very popular with "electroflight" radio-control aero-modeling hobbyists because of their efficiency, power, longevity and light weight in comparison to traditional brushed motors. Brushless AC motor controllers are much more complicated than brushed motor controllers.

The correct phase varies with the motor rotation, which is to be taken into account by the ESC.Usually, back EMF from the motor is used to detect this rotation, but variations exist that use magnetic (Hall Effect) or optical detectors. Computer-programmable [6, 7] speed controls generally have user-specified options which allow setting low voltage cut-off limits, timing, acceleration, braking and direction of rotation. Reversing the motor's direction may also be accomplished by switching any two of the three leads from the ESC to the motor. Electronic Speed Controllers (ESC) are an essential component of modern quadcopters (and all multirotors) that offer high power, high frequency, high resolution 3-phase AC power to the motors in an extremely compact miniature package. These craft depend entirely on the variable speed of the motors driving the propellers. This wide variation and fine RPM control in motor/prop speed gives all of the control necessary for a quadcopter (and all multirotors) to fly.

Height is determined by the amount of power to all four motors. Forward motion is achieved by driving the aft (back) props faster than the forward props. Sideways motion is achieved by running the left or right props faster. 'Rudder' movements (yaw), (turning left or right) are again achieved by slowing or speeding individual motors - and this control is reliant on the fact that two of the rotors rotate clockwise while the other two rotate counterclockwise so that, again, slowing or speeding individual motors (and props) will produce a change in attitude in the craft. Quadcopters are a rapidly growing hobby subject but also provide aerial mounts for video cameras for sports coverage, agricultural research, inspection of electrical pylons and historic exploration. Figure 6 shows the Electronic speed controller.

Quadcopter ESCs usually can use a faster update rate compared to the standard $50 \mathrm{~Hz}$ signal used in most other RC applications. PWM signals up to $400 \mathrm{~Hz}$ can be used in some cases, and other control options can increase this rate even higher. Also, some software delays, such as low-pass filters, are removed in order to improve control latency.

Figure 7 displays the Arduini board. The Arduino Uno is a microcontroller board based on the ATmega328. It has 14 digital input/output pins (of which 6 can be used as PWM outputs), 6 analog inputs, a 
$16 \mathrm{MHz}$ crystal oscillator, a USB connection, a power jack, an ICSP header, and a reset button. Figure 8 shows the PIN diagram of IC ATmega328P. It contains everything needed to support the microcontroller, simply connect it to a computer with a USB cable or power it with a AC-to-DC adapter or battery to get started. The Uno differs from all preceding boards in that it does not use the FTDI USB-to-serial driver chip. Instead, it features the Atmega8U2 programmed as a USB-to-serial converter.

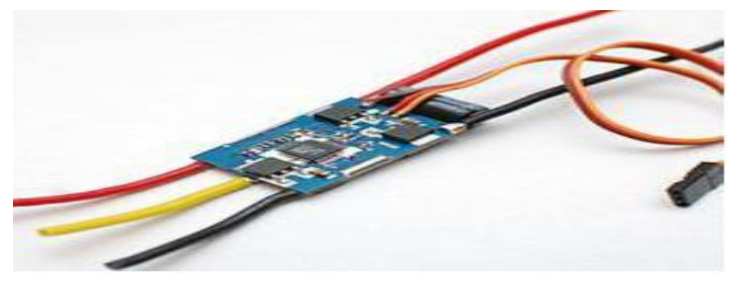

Figure 6. Electronic speed controller

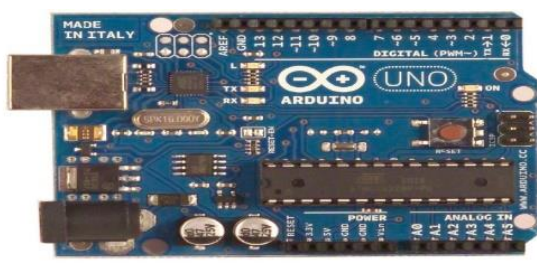

Figure 7. Arduino board

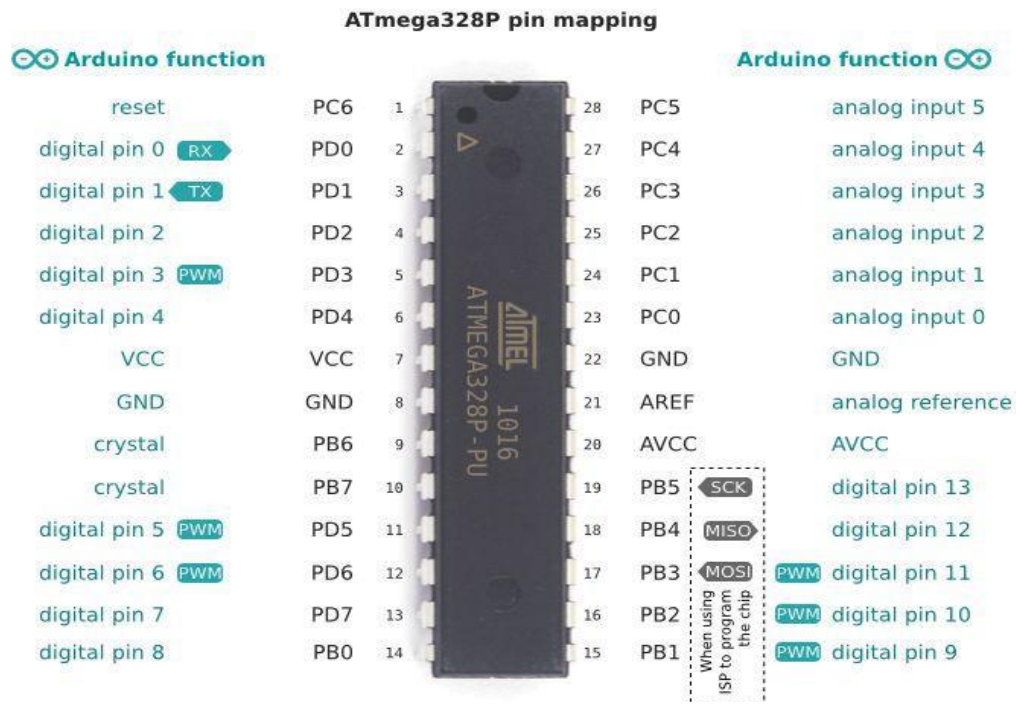

Figure 8. Pin diagram

This mini wireless spy camera Transmitter and Receiver set comes with everything you need for, spy and surveillance right out of the box! You get a tiny 1/3 inch CMOS wireless camera transmitter that captures both video and audio. It can be powered from a $9 \mathrm{~V}$ battery or normal wall socket. Plus you also get a wireless long range receiver which can output the transmitted camera imagery to any standard TV with AV connectors. It's powered from a normal wall socket and comes with a high power antenna for better reception quality. Figure 9 display the wireless camera.

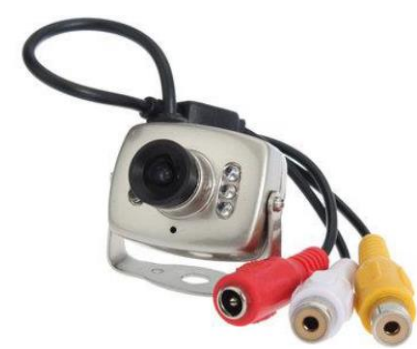

Figure 9. Wireless camera 
Let's say you want to know what gets said in the boardroom when the company directors meet every month. Simply attach a $9 \mathrm{~V}$ battery to the wireless camera transmitter and place it in a in conspicuous corner of the boardroom with the lens aimed at where the action will be. Meanwhile, put yourself another room with the wireless receiver hooked up to a small TV. Everything that gets said and done in the boardroom gets transmitted to your display monitor. And even in the unlikely event that they spot the camera, you don't care-with its low, low price tag. Several types of vehicle tracking devices exist. Typically they are classified as "passive" and "active". "Passive" devices store GPS location, speed, heading and sometimes a trigger event such as key on/off, door open/closed. Once the vehicle returns to a predetermined point, the device is removed and the data downloaded to a computer for evaluation. Passive systems include auto download type that transfer data via wireless download. "Active" devices also collect the same information but usually transmit the data in near-real-time via cellular or satellite networks to a computer or data center for evaluation. Many modern vehicle tracking devices combine both active and passive tracking abilities when a cellular network is available and a tracking device is connected it transmits data to a server; when a network is not available the device stores data in internal memory and will transmit stored data to the server later when the network becomes available again. Figure 10 shows the GPS system. Major constituents of the GPS-based tracking are:

a. GPS tracking: The device fits into the vehicle and captures the GPS location information apart from other vehicle information at regular intervals to a central server. Other vehicle information can include fuel amount, engine temperature, altitude, reverse geocoding, door open/close, tire pressure, cut off fuel, turn off ignition, turn on headlight, turn on taillight, battery status, GSM area code/cell code decoded, number of GPS satellites in view, glass open/close, fuel amount, emergency button status, cumulative idling, computed odometer, engine RPM, throttle position, GPRS status and a lot more. Capability of these devices actually decide the final capability of the whole tracking system; most vehicle tracking systems, in addition to providing the vehicle's location data, feature a wide range of communication ports that can be used to integrate other on board systems, allowing to check their status and control or automate their operation.

b. GPS tracking server: The tracking server has three responsibilities: receiving data from the GPS tracking unit, securely storing it, and serving this information on demand to the user.

c. User interface: The UI determines how one will be able to access information, view vehicle data, and elicit important details from it.GT02 is a exquisite and powerful GPS/GSM/GPRS vehicle tracker, focusing on the features of vehicles. With the characteristics of free maintenance and strong signal strength, it is ultra-thin and easy to install. The built-in high-sensitivity SiRF III GPS chip and antenna grant GT02 the ability of quick positioning even in the metropolitan areas. The user can check its position and route through network or mobile. GT02 activates intelligent working method, which enables remote control and monitor preservation without any operation after installation. It is definitely a maintenance free intelligent positioning terminal.

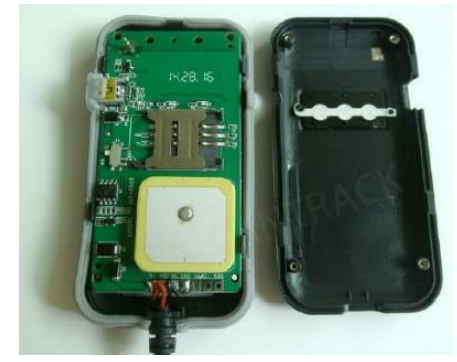

Figure 10. GPS system

Radio control (often abbreviated to R/C or simply RC shown in Figure 11) is the use of radio signals to remotely control a device. Radio control is used for control of model vehicles from a hand-held radio transmitter. Industrial, military, and scientific research organizations make use of radio-controlled vehicles as well. Remote control military applications are typically not radio control in the direct sense, directly operating flight control surfaces and propulsion power settings, but instead take the form of instructions sent to a completely autonomous, computerized automatic pilot. Instead of a "turn left" signal that is applied until the aircraft is flying in the right direction, the system sends a single instruction that says "fly to this point". Some of the most outstanding examples of remote radio control of a vehicle are the Mars Exploration Rovers such as Sojourner. 
The first general use of radio control systems in models started in the early 1950s with singlechannel self-built equipment; commercial equipment came later. The advent of transistors greatly reduced the battery requirements, since the current requirements at low voltage were greatly reduced and the high voltage battery was eliminated. In both tube and early transistor sets the model's control surfaces were usually operated by an electromagnetic escapement controlling the stored energy in a rubber-band loop, allowing simple on/off rudder control (right, left, and neutral) and sometimes other functions such as motor speed. Crystal-controlled super-heterodyne receivers with better selectivity and stability made control equipment more capable and at lower cost. Multi-channel developments were of particular use to aircraft, which really needed a minimum of three control dimensions (yaw, pitch and motor speed), as opposed to boats, which required only two or one. As the electronics revolution took off, single-signal channel circuit design became redundant, and instead radios provided proportionally coded signal streams which a servomechanism could interpret, using pulse-position modulation (PPM). More recently, high-end hobby systems using Pulse-code modulation (PCM) features have come on the market that provide a computerized digital bit-stream signal to the receiving device, instead of the earlier PPM encoding type. However, even with this coding, loss of transmission during flight has become more common, in part because of the ever more wireless society. Some more modern FM-signal receivers that still use "PPM" encoding instead can, thanks to the use of more advanced computer chips in them, be made to lock onto and use the individual signal characteristics of a particular PPM-type RC transmitter's emissions alone, without needing a special "code" transmitted along with the control information as PCM encoding has always required. Figure 12 display the complete prototype and Figure 13 shows the base station.

In the early 21 st century, 2.4 gigahertz spread spectrum RC control systems have become increasingly utilized in control of model vehicles and aircraft. Now, these $2.4 \mathrm{GHz}$ systems are being made by most radio manufacturers. These radio systems range from a couple thousand dollars, all the way down to under US\$30 for some. Some manufacturers even offer conversion kits for older digital $72 \mathrm{MHz}$ receivers and radios. As the emerging multitude of $2.4 \mathrm{GHz}$ band spread spectrum RC systems usually use a "frequency-agile" mode of operations, like FHSS that do not stay on one set frequency any longer while in use, the older "exclusive use" provisions at model flying sites needed for VHF-band RC control systems's frequency control, for VHF-band RC systems that only used one set frequency unless serviced to change it, are not as mandatory as before.

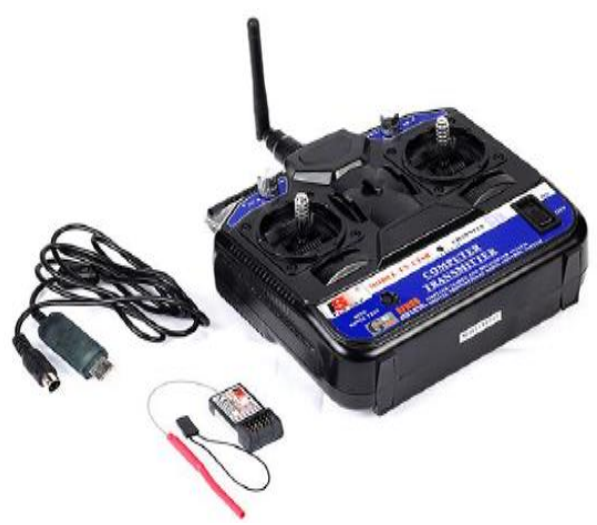

Figure 11. RC transmitter and receiver

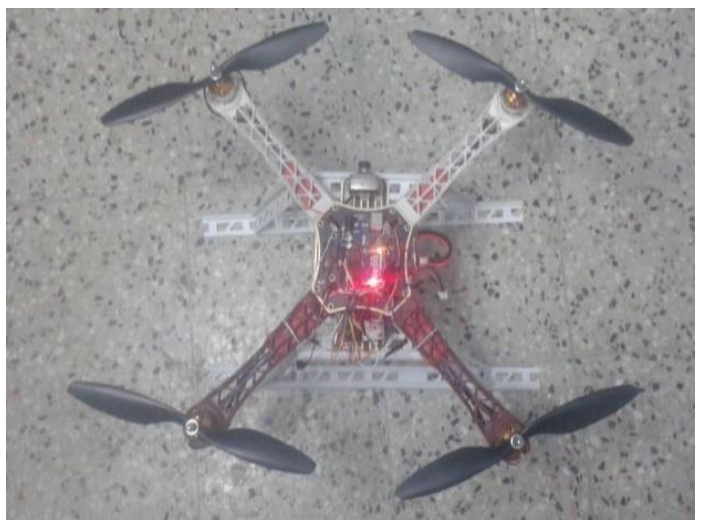

Figure 12. Hardware snapshot (Quadacopter)

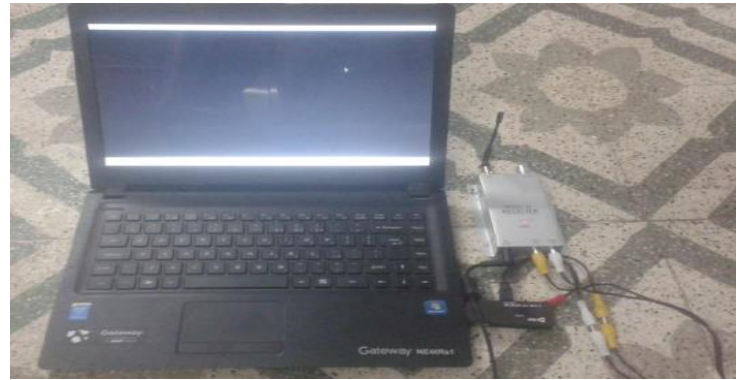

Figure 13. Base station 


\section{SOFTWARE DESCRIPTION}

The Arduino Uno can be programmed with the Arduino software (download). Select "Arduino Uno from the Tools > Board menu (according to the microcontroller on your board). The ATmega328 on the Arduino Uno comes pre burned with a bootloader that allows you to upload new code to it without the use of an external hardware programmer. It communicates using the original STK500 protocol (reference, C header files). You can also bypass the bootloader and program the microcontroller through the ICSP (In-Circuit Serial Programming) header; see these instructions for details. The ATmega16U2 (or 8U2 in the rev1 and rev2 boards) firmware source code is available. The ATmega16U2/8U2 is loaded with a DFU bootloader, which can be activated by:

- On Rev1 boards: connecting the solder jumper on the back of the board (near the map of Italy) and then resetting the $8 \mathrm{U} 2$.

- $\quad$ On Rev2 or later boards: there is a resistor that pulling the 8U2/16U2 HWB line to ground, making it easier to put into DFU mode.

The usage Atmel's FLIP software (Windows) or the DFU programmer (Mac OS X and Linux) to load a new firmware or the usage the ISP header with an external programmer (overwriting the DFU bootloader). This requires the Arduino language 1.0 or higher. There are two recommended software options for using this language. Codebender is a very easy to use, web-browser-based editor for Arduino sketches. With a free account, you can create, share, and run your code from Windows, Mac, Linux, and Chromebook. Arduino software gets installed on your computer, and you do not have to be online to use it. It works with Windows, Mac, and Linux. Figure 14 shows the arduino programming environment. If this is your first time using an Arduino, Activity \#1 will help you get started with your choice of software, connect your hardware, and test your programming connection.

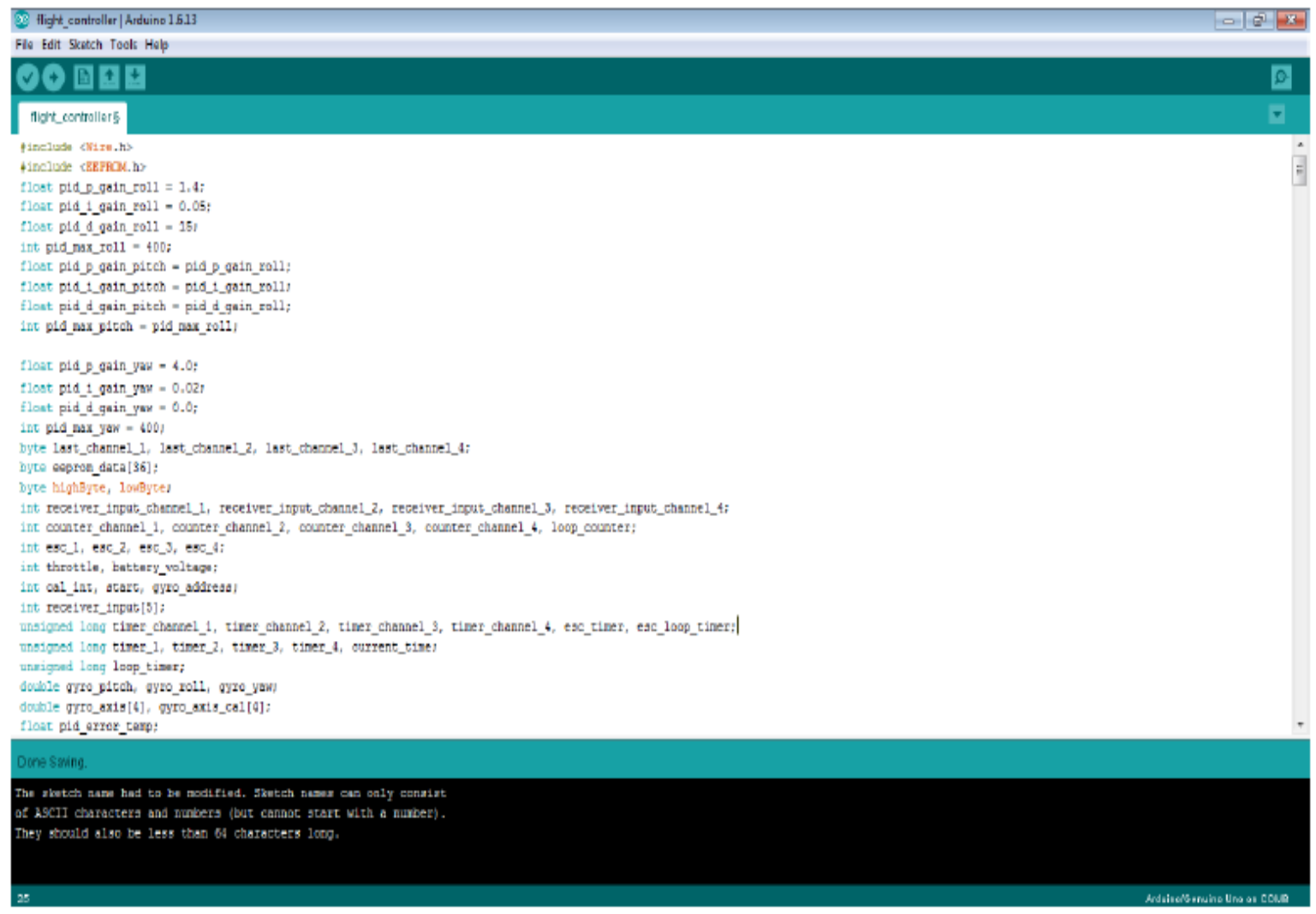

Figure 14. Arduino programming

\section{CONCLUSION}

The quadcopter is an innovative invention in robotics technology presented recently with interesting properties such as stability characteristics and also increasing the thrust .It employs a unique nature low cost control board with high performance and enlarges its application fields. Therefore it has attracted more

Quadcopter based emergency medikit delivery system for hill stations (C. R. Balamurugan) 
attention than the other copters over the last two decades and has already been tried with many applications. Various quadcopter technologies have been reported in the literature. This article presented an intensive applications of the quadcopter frame structures is capable of keeping a stabilized flight while lifting $2 \mathrm{~kg}$ of medicine needed for quadcopters to fly autonomously. Also a top protection was designed to protect the electrical components from rain, dust and other hazards. The proposed electrical system, that included a sensor array,brushless motor speed controllers and other peripherals will allow the cration of feedback loop to control the quadcopter through the system controller. After through validation , the results clearly indicate that the location of the accidental area can be determined by using the GPS module allowing a proper delivary of medikit. In future, ANN controller is capable of controlling the designed quadcopter in ideal conditions, although better results may be attain through the further tuning. In this review, we have discussed an overview of trust management which includes the highlights on semantics of trust, types of trust and attributes used for evaluating trust. Further, we identify the various trust models classified by many researchers and we mainly focused on three trust models namely SLA based, Reputation based and recommendation based trust model. Customers are worried about their data and seeking high confidence level even though a service or provider has a higher trust value. The lack of efficient and reliable trust evaluation system is still a major concern. To improve the efficacy of trust results we can combine reputation and recommender based trust mechanisms in future. New mechanisms may be designed to assess the trusty service provider using fuzzy sets and rough sets.

\section{REFERENCES}

[1] P. Pounds, R. Mahony and J. Gresham, "Towards dynamically-favourable quad-rotor aerial robots," Australian National University, Canberra.

[2] C. Powers, D. Mellinger and V. Kumar, "Quadrotor kinematics and dynamics," in Handbook of Unmanned Aerial Vehicles, New York, Springer Reference, pp. 307 - 328, 2015.

[3] T. Bresciani, "Modelling, identification and control of a quadrotor helicopter," Department of Automatic Control, Lund University, Lund, 2008.

[4] J. Leber, "Doctors without borders is experimenting with delivery drones to battle an epidemic," Fast Company, 2014. [Online]. Available: http://www.fastcoexist.com/3037013/doctors-without-borders-is-experimenting-withdelivery-drones-to-battle-an-epidemic.

[5] D. Hartman, K. Landis, M. Mehrer, S. Moreno, J. Kim, "Quad-sim: Mathematical model documentation," [Online]. Available: http://www.mathworks.com/matlabcentral/fileexchange/48053-quad-sim

[6] Programa de Comunidades Nativas, "La Salud de las Comunidades Nativas: Un reto para el Estado," Informe Defensorial N 134, Defensoría del Pueblo, Lima - Perú, 2008.

[7] Dirección Nacional de Censos y Encuestas, "II Censo de Comunidades Indígenas de la Amazonía Peruana 2007," Tomo 1, Instituto Nacional de Estadística e Informática (INEI), Lima-Perú, 2008. 